Enferm Bras 2019;18(2):242-53

https://doi.org/10.33233/eb.v18i2.2814

\title{
ARTIGO ORIGINAL \\ Nascer de cesárea em microrregião paulista: associação com características sociodemográficas
}

\author{
Luciana Braz de Oliveira Paes, M.Sc. ${ }^{*}$ Zaida Aurora Sperli Geraldes Soler** \\ *Enfermeira Obstétrica, Docente do Centro Universitário Padre Albino (UNIFIPA), Catanduva/SP, \\ ${ }^{*}$ Obstetriz, enfermeira, livre-docente em enfermagem obstétrica, docente e orientadora de \\ graduação e pós-graduação lato sensu e stricto sensu da FAMERP, Orientadora
}

Recebido em 28 de março de 2019; aceito em 29 de abril de 2019.

Endereço para correspondência: Luciana Braz de Oliveira Paes, Rua Barão do Rio Branco, 436 Centro 15828-000 Palmares Paulista SP, E-mail: lucianabrazenf@gmail.com; Zaida Aurora Sperli Geraldes Soler: zaidaaurora@gmail.com

\section{Resumo}

Objetivo: Verificar a associação entre a resolução do parto por cesariana com características sociodemográficas de mulheres que tiveram o parto realizado em um município do interior paulista, no ano de 2012. Métodos: Estudo quantitativo, descritivo, transversal, vinculado ao Projeto-mãe "Estudos sobre o processo do nascimento na Divisão Regional de Saúde XV (DRSXV)", com dados obtidos junto ao Sistema de Informações de Nascidos Vivos (SINASC), aplicando-se o teste qui-quadrado na análise estatística, com significância de 5\%. Resultados: Ocorreram 2846 partos de nascidos vivos em 2012, na cidade estudada, a maioria por cesariana (2396 - 84,2\%). A maioria das mulheres submetidas à cesárea tinha: idade entre 21 a 30 anos (53,7\%); escolaridade de 8 a 11 anos (65,9\%); situação conjugal com companheiro $(66,4 \%)$; fizeram sete ou mais consultas de pré-natal $(87,8 \%)$ e a etnia não estava informada para $68,4 \%$ das mulheres, seguindo-se de $24,3 \%$ de brancas. Houve significância estatística na realização de cesárea entre mulheres com maior idade, maior escolaridade, ter companheiro e fazer mais consultas de pré-natal. Conclusão: As características sociodemográficas interferem no tipo de parto, revelando-se como fator predisponente para realização da cesariana entre mulheres de maior classe social.

Palavras-chave: cesárea, tocologia, parto normal, parto humanizado, humanização da assistência.

\section{Abstract \\ C-section deliveries in a micro-region of São Paulo: association with sociodemographic characteristics}

Objective: To investigate the association between C-section births and the sociodemographic characteristics of women who delivered in a city in the countryside of São Paulo in year 2012. Methods: This quantitative, descriptive, cross-sectional study is linked to a main project called "Studies on the childbirth process in the city and region of São José do Rio Preto/SP (DRS XV)". Data were obtained from the SINASC (Information System on Live Borns or Sistema de Informações de Nascidos Vivos, in Portuguese). The data were subjected to chi-square analysis at the $5 \%$ significance level. Results: There were 2,846 live births in 2012 in the study city and most of them were C-sections $(2,396-84.2 \%)$. Most of the women who were subjected to Csection were: aged $21-30$ years (53.7\%); had 8-11 years of schooling (65.9\%); had the marital status 'with a partner' (66.4\%); and had attended seven or more prenatal consultations $(87.8 \%)$. In this sample, $68.4 \%$ did not report their ethnicity and $24.3 \%$ were white. We found a statistically significant difference in C-section occurrence between women with greater age and education, who had a partner, and attended a greater number of prenatal consultations and women who did not. Conclusion: Sociodemographic characteristics influence childbirth delivery method and prove to be a predisposing factor for cesarean section among women of higher social class.

Key-words: cesarean section, midwifery, natural childbirth, humanizing delivery, humanization of assistance. 


\section{Resumen \\ El nacer por cesárea en una micro-región de São Paulo: asociación con características sociodemográficas}

Objetivo: Investigar la asociación entre resolución de parto por cesárea y características sociodemográficas de mujeres que dieron a luz en un municipio del interior de São Paulo en el año 2012. Métodos: Estudio cuantitativo, descriptivo de corte transversal, vinculado al proyecto matriz «Estudios sobre el proceso del nacimiento en la División Regional de Salud XV (DRS XV)». Los datos se obtuvieron en el SINAC (Sistema de Informaciones de Nacidos Vivos). En el análisis estadístico, se aplicó el test de chi-cuadrado, con un nivel de significancia de 5\%. Resultados: Ocurrieron 2846 partos de nacidos vivos en 2012 en la ciudad estudiada, la mayor parte por cesárea $(2396-84,2 \%)$. La mayoría de las mujeres sometidas a cesárea tenía: edad entre 21 y 30 años (53,7\%); escolaridad entre 8 y 11 años $(65,9 \%)$; situación matrimonial con pareja (66,4\%); siete o más consultas prenatales (87,8\%); y etnicidad no informada (68,4\%). El $24,3 \%$ eran blancas. Hubo significancia estadística en la realización de cesárea entre mujeres de mayor edad y escolaridad, con pareja y un mayor número de consultas prenatales. Conclusión: Las características sociodemográficas influencian el tipo de parto, revelándose como un factor predisponente para la realización de cesárea entre mujeres de clase social más alta.

Palabras-clave: cesárea, tocología, parto normal, parto humanizado, humanización de la atención.

Introdução

Um indicador internacionalmente reconhecido na qualidade da assistência obstétrica é a taxa de cesariana [1]. Em 1985, a Organização Mundial da Saúde publicou uma primeira referência para taxas de cesariana, enfatizando que não há justificativa para que em qualquer país, estado, região, município ou instituição de saúde, os índices sejam superiores a 10-15\% [2].

Recentemente, pela Portaria № 306, de 28 de março de 2016, foram aprovadas as "Diretrizes de Atenção à Gestante: a operação cesariana", ficando em destaque que para definir a taxa de cesariana no Brasil devem ser consideradas as características da nossa população, que apresenta um elevado número de operações cesarianas anteriores, sendo ajustada pela OMS uma taxa entre $25 \%-30 \%$ [3].

Em muitas regiões brasileiras não temos tais taxas de partos normais, sendo aventado que são muitas as razões para o aumento progressivo das cesarianas no Brasil: a escolha das mulheres; a decisão do médico; as influências culturais e socioeconômicas; fatores obstétricos; falhas na assistência no pré-natal e o pagamento, sugerindo que o parto cirúrgico é um bem de consumo [3-6].

O nascimento hospitalar adotou tecnologias para torná-lo mais seguro, com objetivo de diminuir a mortalidade materna e perinatal, mas, por outro lado, permitiu a concretização de um modelo que considera como doença o ciclo gravídico-puerperal e não como expressões de saúde. No entanto, há uma alta incidência de mortalidade materna, pois todos os anos morrem no mundo inteiro, aproximadamente 287 mil mulheres, devido a complicações relacionadas à maternidade [3,7].

Uma das grandes lacunas da assistência à saúde no Brasil é a ausência de trabalho em equipe que proporcione melhoria dos indicadores na assistência perinatal $[8,9]$. Em todo o mundo são divulgadas pesquisas que revelam como evidências científicas que a condução do trabalho de parto por enfermeiras obstétricas e obstetrizes, aumentam as chances de partos espontâneos e diminuem as intervenções desnecessárias, sem comprometer a saúde das mulheres e dos bebês $[8,9]$.

Isso contrasta com o que se vê no Brasil, já que só $15 \%$ dos nascimentos foram assistidos por enfermeiros, enfermeiros obstetras/obstetrizes, com mais frequência nas regiões mais pobres, onde quase não há médicos [11-12].

Incontestavelmente, nas situações de risco materno-fetal, o parto cirúrgico constituiu um avanço da medicina técnico científica. Porém, o que está sendo aqui problematizado é o quanto a indicação de cesariana está atendendo a interesses médicos [13]. Causa indignação no Brasil a realização de cesarianas por motivos ilegítimos, a omissão de informações no ciclo gravídicopuerperal, o desrespeito pelos sentimentos e necessidades das mulheres e famílias, fatos que revelam formas disfarçadas de violência obstétrica $[4,13,14]$. 
Ante o exposto, este estudo teve como objetivo verificar a associação entre a cesárea com as características sociodemográficas de mulheres que tiveram o parto realizado em 2012 , em um município do interior paulista.

\section{Material e métodos}

Este estudo é descritivo, transversal, com abordagem quantitativa, realizado em uma cidade incluída na microrregião da Secretaria de Estado da Saúde de São Paulo - DRSXV, referência de atendimento para 19 municípios.

A coleta de dados foi realizada através do SINASC (Sistema de Informações de Nascidos Vivos). As variáveis avaliadas foram o tipo de parto; faixa etária da mãe, escolaridade, número de consultas no pré-natal, situação civil, etnia, peso do RN e Apgar. Os dados foram coletados no ano de 2016, considerando-se os nascimentos ocorridos de janeiro a dezembro de 2012, correspondendo ao ano com maiores índices de cesarianas entre 2006 e 2014.

Esta pesquisa inclui-se no projeto-mãe "Estudos sobre o processo do nascimento na Divisão Regional de Saúde XV (DRSXV)". Vale esclarecer, ademais, que esta pesquisa dispensa aprovação de Comitê de Ética, conforme resoluções 196/1996 e 466/2012 do sistema CEP/CONEP, pois o SINASC é uma base de dados governamental, de domínio público e não identifica os participantes.

Foi aplicado o teste qui-quadrado nas análises, com o objetivo de observar associações entre as variáveis definidas. Os testes estatísticos foram aplicados com significância de $5 \%$ $(\mathrm{P}<0,05)$, utilizando-se o software Minitab 17 (Minitab Inc.). Agruparam-se os dados segundo as variáveis do objetivo da pesquisa e os resultados são apresentados em Tabelas.

\section{Resultados}

O tipo de parto foi relacionado às seguintes variáveis: faixa etária da mãe, escolaridade, número de consultas no pré-natal, situação civil, etnia, peso do RN e nota Apgar.

\section{Caracterização amostral}

Verifica-se na Tabela I: associação significativa em quase todas as relações observadas, porém somente a associação do tipo de parto e etnia da mãe ( $P=0,417)$ não resultou significativa; maior número de cesáreas entre as mães com idade entre 21 e 30 anos $(53,7 \%)$; escolaridade entre 8 a 11 anos (65,2\%); 7 a mais consultas de pré-natal $(85,3 \%)$ e com situação civil com companheiro (64\%). Para $68,4 \%$ das mulheres não estava anotada a etnia, seguindo-se de $24,3 \%$ de mulheres brancas submetidas à cesariana.

No que se refere à relação da escolaridade com a situação civil, foi possível observar relação significativa $(P<0,001)$ entre as mulheres avaliadas que, independentemente de apresentarem ou não companheiro, tinham escolaridade de 8 a 11 anos. Entretanto, os dados mostram certa tendência em sugerir que há quantidade significativa de mães com companheiro que apresentam escolaridade de 12 anos ou mais e que as mães sem companheiro apresentam escolaridade de 4 a 7 anos. O resultado se repete para etnia $(P<0,001)$, pois a maioria das mães apresenta escolaridade de 8 a 11 anos; entretanto, há certa tendência em sugerir que mães de etnia branca apresentam escolaridade de 12 anos ou mais, ao passo que mães de etnia não branca apresentam escolaridade de 4 a 7 anos. 
Tabela I - Associação do tipo de parto com variáveis sociodemográficas e obstétricas maternas. Município da DRSXV, 2012.

\begin{tabular}{|c|c|c|c|c|c|c|c|c|}
\hline \multirow{3}{*}{ Faixa etária } & \multicolumn{8}{|c|}{ Tipo de parto } \\
\hline & \multicolumn{2}{|c|}{ Vaginal } & \multicolumn{2}{|c|}{ Cesáreo } & \multicolumn{2}{|c|}{ Não informado } & \multicolumn{2}{|l|}{ Total } \\
\hline & $\mathrm{N}$ & $\%$ & $\mathrm{~N}$ & $\%$ & $\mathrm{~N}$ & $\%$ & $\mathrm{~N}$ & $\%$ \\
\hline 10 a 14 anos & 2 & 0,4 & 13 & 0,5 & 0 & 0,0 & 15 & 0,5 \\
\hline 15 a 20 anos & 147 & 32,9 & 418 & 17,4 & 1 & 0,03 & 566 & 19,9 \\
\hline 21 a 30 anos & 223 & 49,9 & 1286 & 53,7 & 1 & 0,03 & 1510 & 53,1 \\
\hline 31 a 40 anos & 73 & 16,3 & 656 & 27,4 & 1 & 0,03 & 730 & 25,7 \\
\hline 41 a 50 anos & 2 & 0,4 & 23 & 1,0 & 0 & 0,0 & 25 & 0,9 \\
\hline Total & 447 & 15,7 & 2396 & 84,2 & 3 & 0,1 & 2846 & 100 \\
\hline Valor $\mathrm{P}^{1}$ & \multicolumn{8}{|c|}{$<0,001$} \\
\hline \multicolumn{9}{|l|}{ Escolaridade } \\
\hline Nenhuma & 5 & 1,1 & 37 & 1,5 & 0 & 0,0 & 42 & 1,5 \\
\hline 1 a 3 anos & 19 & 4,3 & 42 & 1,8 & 0 & 0,0 & 61 & 2,1 \\
\hline 4 a 7 anos & 128 & 28,6 & 317 & 13,2 & 0 & 0,0 & 445 & 15,6 \\
\hline 8 a 11 anos & 273 & 61,1 & 1580 & 65,9 & 2 & 0,07 & 1855 & 65,2 \\
\hline 12 anos ou mais & 22 & 4,9 & 420 & 17,5 & 1 & 0,03 & 443 & 15,6 \\
\hline Total & 447 & 15,7 & 2396 & 84,2 & 3 & 0,1 & 2846 & 100 \\
\hline Valor $\mathrm{P}^{1}$ & \multicolumn{8}{|c|}{$<0,001$} \\
\hline \multicolumn{9}{|c|}{ Consultas de Pré-natal } \\
\hline Nenhuma & 14 & 3,1 & 17 & 0,7 & 0 & 0,0 & 31 & 1,1 \\
\hline 1 a 3 & 25 & 5,6 & 43 & 1,8 & 0 & 0,0 & 68 & 2,4 \\
\hline 4 a 6 & 86 & 19,2 & 232 & 9,7 & 0 & 0,0 & 318 & 11,2 \\
\hline 7 ou mais & 322 & 72,0 & 2104 & 87,8 & 3 & 0,1 & 2429 & 85,3 \\
\hline Total & 447 & 15,7 & 2396 & 84,2 & 3 & 0,1 & 2846 & 100 \\
\hline Valor $\mathrm{P}^{1}$ & \multicolumn{8}{|c|}{$<0,001$} \\
\hline \multicolumn{9}{|l|}{ Situação civil } \\
\hline Com companheiro & 228 & 51,0 & 1592 & 66,4 & 2 & 0,07 & 1822 & 64,0 \\
\hline Sem companheiro & 217 & 48,5 & 794 & 33,1 & 1 & 0,03 & 1012 & 35,6 \\
\hline Não informado & 2 & 0,4 & 10 & 0,4 & 0 & 0,0 & 12 & 0,4 \\
\hline Total & 447 & 15,7 & 2396 & 84,2 & 3 & 0,1 & 2846 & 100 \\
\hline Valor $\mathrm{P}^{1}$ & \multicolumn{8}{|c|}{$<0,001$} \\
\hline \multicolumn{9}{|l|}{ Etnia } \\
\hline Branca & 103 & 23,0 & 582 & 24,3 & 0 & 0,0 & 685 & 24,1 \\
\hline Não branca & 37 & 8,3 & 176 & 7,3 & 0 & 0,0 & 213 & 7,5 \\
\hline Não informada & 307 & 68,7 & 1638 & 68,4 & 3 & 0,1 & 1948 & 68,4 \\
\hline Total & 447 & 15,7 & 2396 & 84,2 & 3 & 0,1 & 2846 & 100 \\
\hline Valor $\mathrm{P}^{1}$ & \multicolumn{8}{|c|}{0,417} \\
\hline
\end{tabular}

${ }^{1}$ Valor $\mathrm{P}$ referente ao teste qui-quadrado a $\mathrm{P}<0,05$.

Nas Tabelas II a IV apresenta-se apenas os 2396 partos cesarianos realizados em 2012 no município estudado, analisando-se algumas variáveis sociodemográficas maternas e do neonato associadas.

Idade gestacional

A idade gestacional foi relacionada com algumas variáveis a fim de observar a presença de associações com peso da criança ao nascer, escolaridade, faixa etária da mãe, profissão e número de consultas pré-natal (Tabela II), destacando-se:

- Associação significativa entre a idade gestacional e escolaridade $(P<0,001)$;

- A maioria das mães, independentemente da idade gestacional, tinha de oito a 11 anos de escolaridade (1580- 65,9\%);

- Não houve associação significativa entre a idade gestacional e a faixa etária da mãe $P=0,175$;

- A maioria das mães com idade gestacional de 22 a 27 semanas realizou de 4 a 6 consultas $(53,8 \%)$; 
- A maioria das mães com idade gestacional acima de 27 semanas realizou 7 ou mais consultas de pré-natal $(2103-87,8 \%)$;

- Entre as mães com idade gestacional de 28 a 31 semanas, $17(51,5 \%)$ realizaram 7 ou mais consultas de pré-natal e $13(39,4 \%)$ realizaram de 4 a 6 consultas

Tabela II - Relação da idade gestacional com variáveis sociodemográficas de mulheres que realizaram cesárea. Município da DRSXV, 2012.

\begin{tabular}{|c|c|c|c|c|c|c|c|c|c|c|c|}
\hline \multirow{3}{*}{$\begin{array}{l}\text { Escolaridade } \\
\text { (anos) }\end{array}$} & \multicolumn{11}{|c|}{ Idade gestacional (semanas) } \\
\hline & \multirow{2}{*}{$\begin{array}{l}22 / 27 \\
\mathrm{~N}\end{array}$} & \multicolumn{2}{|c|}{$28 / 31$} & \multicolumn{2}{|c|}{32 a 36} & \multicolumn{2}{|c|}{37 a 41} & $42+$ & $\begin{array}{l}\text { Não } \\
\text { informado }\end{array}$ & \multicolumn{2}{|l|}{ Total } \\
\hline & & $\mathrm{N}$ & & $\mathrm{N}$ & & $\mathrm{N}$ & & $\mathrm{N}$ & $\mathrm{N}$ & $\mathrm{N}$ & $\%$ \\
\hline $\begin{array}{l}\text { Não } \\
\text { informado }\end{array}$ & 0 & 1 & & 3 & & 26 & & 0 & 0 & 30 & 1,3 \\
\hline Nenhuma & 0 & 1 & & 3 & & 3 & & 0 & 0 & 7 & 0,3 \\
\hline 1 a 3 & 0 & 0 & & 14 & & 28 & & 0 & 0 & 42 & 1,8 \\
\hline 4 a 7 & 2 & 7 & & 51 & & 254 & & 2 & 1 & 317 & 13,2 \\
\hline 8 a 11 & 11 & 19 & & 185 & & 1358 & & 3 & 4 & 1580 & 65,9 \\
\hline 12 ou mais & 0 & 5 & & 49 & & 366 & & 0 & 0 & 420 & 17,5 \\
\hline Total & & 33 & & 305 & & 2035 & & 5 & 5 & 2396 & 100 \\
\hline Valor $\mathrm{P}^{1}$ & $<0,001$ & & & & & & & & & & \\
\hline \multicolumn{12}{|l|}{$\begin{array}{l}\text { Faixa etária } \\
\text { (anos) }\end{array}$} \\
\hline 10 a 14 & 0 & & 0 & & 5 & & 8 & $c$ & 0 & 13 & 0,5 \\
\hline 15 a 20 & 0 & & 2 & & 61 & & 352 & 1 & 2 & 418 & 17,4 \\
\hline 21 a 30 & 11 & & 22 & & 153 & & 1096 & 2 & 2 & 1286 & 53,7 \\
\hline 31 a 40 & 2 & & 8 & & 84 & & 559 & 2 & 1 & 656 & 27,4 \\
\hline 41 a 50 & 0 & & 1 & & 2 & & 20 & c & 0 & 23 & 1,0 \\
\hline Total & & & 33 & & 305 & & 2035 & 5 & 5 & 2396 & 100 \\
\hline Valor $\mathrm{P}^{1}$ & 0,175 & & & & & & & & & & \\
\hline \multicolumn{12}{|l|}{$\begin{array}{l}\text { № consultas } \\
\text { pré-natal }\end{array}$} \\
\hline Nenhuma & 0 & & 0 & & 3 & & 10 & $c$ & 1 & 14 & 0,6 \\
\hline 1 a 3 & 5 & & 2 & & 13 & & 22 & $c$ & 1 & 43 & 1,8 \\
\hline 4 a 6 & 7 & & 13 & & 69 & & 140 & 2 & 1 & 232 & 9,7 \\
\hline 7 ou mais & 1 & & 17 & & 220 & & 1861 & 3 & 2 & 2104 & 87,8 \\
\hline $\begin{array}{l}\text { Não } \\
\text { informado }\end{array}$ & 0 & & 1 & & 0 & & 2 & $\mathrm{c}$ & $\overline{0}$ & 3 & 0,1 \\
\hline Total & & & 33 & & 305 & & 2035 & 5 & 5 & 2396 & 100 \\
\hline Valor $\mathrm{P}^{1}$ & $<0,001$ & & & & & & & & & & \\
\hline
\end{tabular}

Apresenta-se a seguir a associação entre peso do recém-nascido e variáveis sociodemográficas maternas (Tabela III).

Peso ao nascer

Os resultados indicam (tabela III) associação significativa entre a idade gestacional e 0 peso da criança ao nascer $(\mathrm{P}<0,001)$. De acordo com os resultados obtidos, quanto menor a idade gestacional, menor será o peso do recém-nascido. Verifica-se também na Tabela III:

- Independentemente do peso do recém-nascido, ocorreu maior frequência de nascimentos com mães pertencentes à faixa etária de 21 a 30 anos;

- O percentual de ocorrência de nascimento foi significativamente superior para os recém-nascidos $(\mathrm{RN})$ com peso superior a $1000 \mathrm{~g}$ ocorreram mais em mães com faixa etária de 21 a 40 anos;

- $\quad \mathrm{Na}$ associação entre o peso do RN e o número de consultas no pré-natal, a maioria das mães que deram à luz ao RN com peso inferior a $1000 \mathrm{~g}$, a ocorrência de 4 a 6 consultas foi superior em relação às mães que deram à luz aos $\mathrm{RN}$ com peso superior 
a 1000 g. Existe uma certa tendência em sugerir que quanto menor o peso do RN menor será o número de consultas no pré-natal;

- Na associação do peso do RN com o escore Apgar no 1ํ e 5은 minutos, o resultado é semelhante para ambos os casos; ou seja, o escore Apgar 8 a 10 (maior escore) excede os $70 \%$ a partir do peso do RN acima de $1500 \mathrm{~g}$, excedendo $90 \%$ de ocorrência acima de $2500 \mathrm{~g}$. No entanto, se o RN apresentar peso abaixo de $1500 \mathrm{~g}$, a probabilidade de apresentar escores baixos de Apgar é maior quando comparado aos $\mathrm{RN}$ com peso superior a $1500 \mathrm{~g}$. Sendo assim, quanto menor o peso do RN, menor será o seu escore Apgar tanto no $1^{\circ} \stackrel{-}{\text { quanto }}$ no $5^{\circ}$ minuto.

Tabela III - Associação do peso do recém-nascido com faixa etária da mãe, número de consultas pré-natal e escore Apgar de mulheres e recém-nascidos, com nascimento por cesárea. Município da DRSXV, 2012.

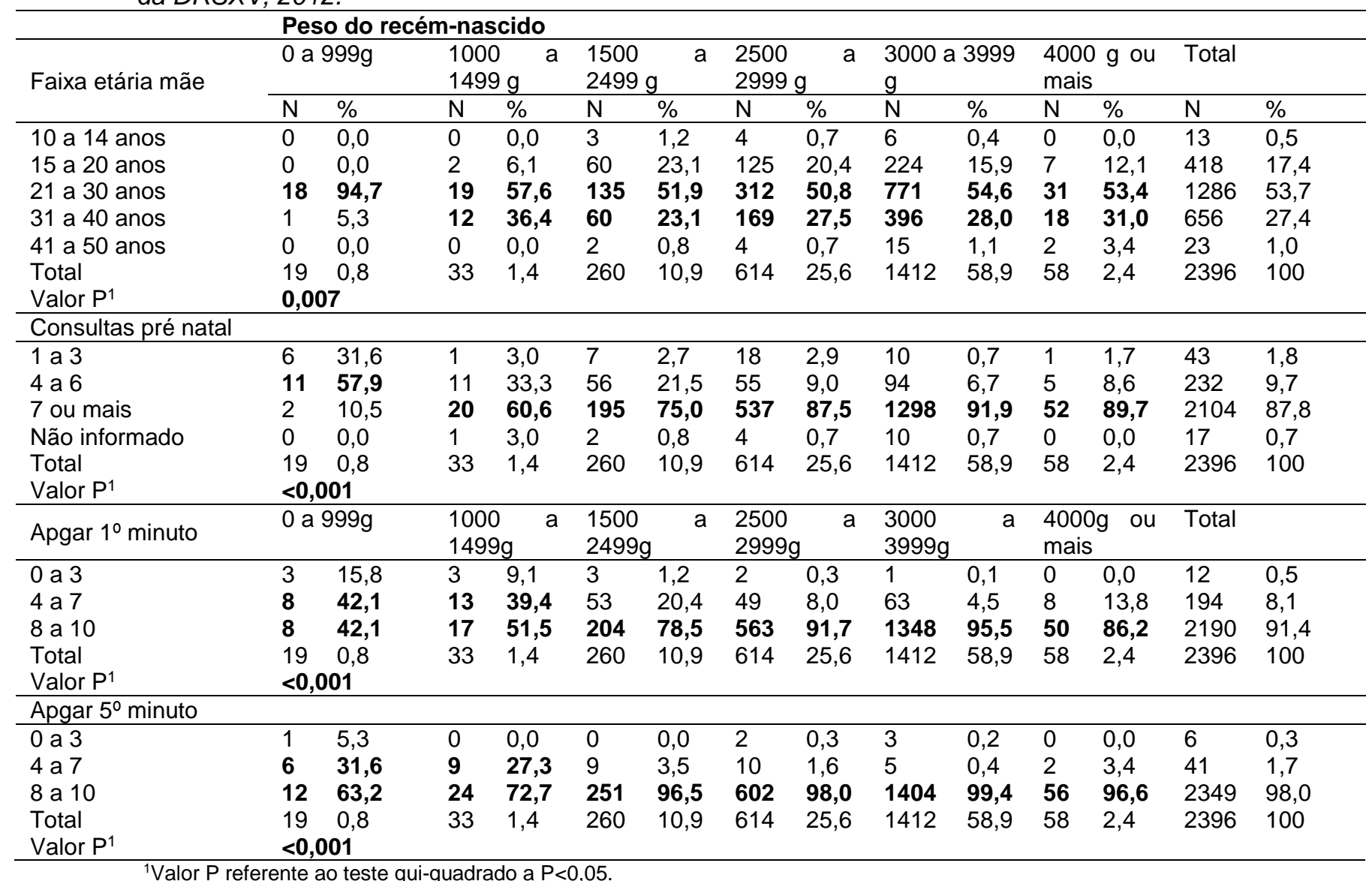

Número de consultas no pré-natal

O número de consultas no pré-natal foi associado com a faixa etária da mãe, escolaridade da mãe e com os escores Apgar no $1^{\circ}$ e $5^{\circ}$ minutos (Tabela IV), verificando-se nesta tabela:

- Elevada associação entre o número de consultas no pré-natal e a faixa etária da mãe, sendo que, na maioria dos casos, independentemente da faixa etária, mais mães realizaram 7 ou mais consultas;

- É possível observar um percentual significativo de ocorrência de 4 a 6 consultas para mães com faixa etária até 14 anos. Esse resultado indica que quanto menor a idade da mãe, menor foi o número de consultas no período pré-natal;

- A associação do número de consultas com a escolaridade também foi significativa, mostrando que para todos os níveis de escolaridade, 7 ou mais consultas foram realizadas de forma mais frequente; 
- É possível observar um elevado percentual de mães com nenhuma escolaridade que realizaram um número inferior de consultas (4 a 6 ). Sendo assim, sugere-se que a falta de escolaridade pode estar associada com o menor número de consultas pré-natal;

- Os resultados da associação entre o número de consultas no período pré-natal e os escores Apgar no $1^{\circ}$ e $5^{\circ}$ minuto mostraram que quanto maior o número de consultas, maior o escore Apgar. Existe certa tendência em sugerir que quanto menor o número de consultas pré-natal, menor será o escore Apgar. Sendo assim, o pré-natal é um fator preponderante para que o RN apresente boas condições ao nascer.

Tabela IV - Associação do número de consultas de pré-natal com alguns dados sociodemográficos das mães e dos neonatos, em nascimentos ocorridos por cesárea.

\begin{tabular}{|c|c|c|c|c|c|c|c|}
\hline \multirow{3}{*}{\multicolumn{2}{|c|}{ Faixa etária mãe }} & \multicolumn{6}{|c|}{ № de consultas no pré-natal } \\
\hline & & \multirow{2}{*}{$\begin{array}{l}\text { Nenhuma } \\
\mathrm{N}\end{array}$} & \multirow{2}{*}{$\begin{array}{l}1 \mathrm{a} 3 \\
\mathrm{~N}\end{array}$} & \multirow{2}{*}{$\frac{4 \text { a } 6}{N}$} & \multirow{2}{*}{$\frac{7 \text { ou mais }}{\mathrm{N}}$} & \multicolumn{2}{|l|}{ Total } \\
\hline & & & & & & $\mathrm{N}$ & $\%$ \\
\hline 10 a 14 & & 1 & 0 & 3 & 9 & 13 & 0,5 \\
\hline 15 a 20 & & 3 & 5 & 55 & 355 & 418 & 17,4 \\
\hline 21 a 30 & & 9 & 25 & 133 & 1119 & 1286 & 53,7 \\
\hline 31 a 40 & & 4 & 13 & 40 & 599 & 656 & 27,4 \\
\hline 41 a 50 & & 0 & 0 & 1 & 22 & 23 & 1,0 \\
\hline Total & & 17 & 43 & 232 & 2104 & 2396 & 100 \\
\hline Valor $\mathrm{P}^{1}$ & & 0,002 & & & & & \\
\hline \multicolumn{8}{|l|}{ Escolaridade } \\
\hline Não informado & & 0 & 0 & 4 & 26 & 30 & 1,3 \\
\hline Nenhuma & & 0 & 0 & 3 & 4 & 7 & 0,3 \\
\hline 1 a 3 anos & & 3 & 2 & 8 & 29 & 42 & 1,8 \\
\hline 4 a 7 anos & & 5 & 12 & 58 & 242 & 317 & 13,2 \\
\hline 8 a 11 anos & & 8 & 25 & 148 & 1399 & 1580 & 65,9 \\
\hline 12 anos ou mais & & 1 & 4 & 11 & 404 & 420 & 17,5 \\
\hline Total & & 17 & 43 & 232 & 2104 & 2396 & 100 \\
\hline Valor $\mathrm{P}^{1}$ & & $<0,001$ & & & & & \\
\hline \multicolumn{8}{|l|}{ Apgar1 ${ }^{\circ}$ minuto } \\
\hline 0 a 3 & & 1 & 3 & 3 & 5 & 12 & 0,5 \\
\hline 4 a 7 & & 0 & 2 & 34 & 158 & 194 & 8,1 \\
\hline 8 a 10 & & 16 & 38 & 195 & 1941 & 2190 & 91,4 \\
\hline Total & & 17 & 43 & 232 & 2104 & 2396 & 100 \\
\hline Valor $\mathrm{P}^{1}$ & & $<0,001$ & & & & & \\
\hline \multicolumn{8}{|l|}{ Apgar 5o minuto } \\
\hline 0 a 3 & & 0 & 1 & 1 & 4 & 6 & 0,3 \\
\hline 4 a 7 & & 1 & 2 & 14 & 24 & 41 & 1,7 \\
\hline 8 a 10 & & 16 & 40 & 217 & 2076 & 2349 & 98,0 \\
\hline Total & & 17 & 43 & 232 & 2104 & 2396 & 100 \\
\hline Valor $\mathrm{P}^{1}$ & & $<0,001$ & & & & & \\
\hline
\end{tabular}

${ }^{1}$ Valor $\mathrm{P}$ referente ao teste qui-quadrado a $\mathrm{P}<0,05$.

O tipo de parto foi relacionado com algumas das variáveis estudadas, sendo elas: faixa etária da mãe, escolaridade, número de consultas no pré-natal, situação civil, etnia, peso do RN e escore Apgar (Tabela V).

Tipo de parto

Os resultados da Tabela $V$ indicam a presença de associação significativa em quase todas as relações observadas, mas somente a relação do tipo de parto e etnia da mãe $(P=0,417)$ não resultou significativa. Verifica-se também na Tabela V:

- A relação entre o tipo de parto e a faixa etária da mãe mostrou maior ocorrência de parto cesáreo de mães com idade entre 21 e 30 anos; 
- Há certo equilíbrio entre a idade das mães que realizaram parto vaginal, com idade entre 15 e 30 anos. Sendo assim, há certa tendência em sugerir que mães mais jovens optem por parto vaginal e mães com idade superior optem pelo parto cesáreo;

- Na relação do tipo de parto com escolaridade, em ambos os casos de parto vaginal ou cesáreo, a maioria das mães apresentou escolaridade de 8 a 11 anos. Há certa tendência em sugerir que mães com escolaridade inferior ( 4 a 7 anos) optem pelo parto vaginal e mães com escolaridade superior (12 anos ou mais) optem pelo parto cesáreo;

- Em relação ao número de consultas, o resultado é semelhante, ou seja, em ambos os casos, as mães realizaram 7 ou mais consultas no pré-natal. Há uma tendência em considerar que mães que realizaram de 4 a 6 consultas optassem pelo parto vaginal, de forma que o parto normal pode estar associado ao menor número de consultas no pré-natal;

- No que se refere à associação do tipo de parto com a situação civil, a grande maioria das mães casadas ou em união estável optou pelo parto cesáreo, ao passo que há certo equilíbrio na opção pelo partos vaginal e cesáreo pelas mães que não tinham companheiro. Sendo assim, a falta de um companheiro pode influenciar de forma significativa no tipo de parto, considerando, nesse caso, uma tendência pela opção ao parto vaginal;

- O peso do RN também foi associado de forma significativa com o tipo de parto $(P=0,024)$, observando-se que quanto menor o peso do $R N$, maior foi a frequência de parto vaginal. Na maioria dos partos, os recém-nascidos com peso acima de $1000 \mathrm{~g}$ mostraram certa tendência pelo parto cesáreo, contabilizando mais de $80 \%$ dos casos avaliados neste estudo;

- Tanto o escore do 1 minuto como o do 5 minuto foram superiores para os RN nascidos de parto cesáreo. 
Tabela V - Associação do tipo de parto com algumas variáveis maternas e do neonato, segundo tipo de parto. Município da DRSXV, 2012.

\begin{tabular}{|c|c|c|c|c|c|}
\hline \multirow{2}{*}{$\begin{array}{l}\text { Faixa etária da mãe } \\
\text { (anos) }\end{array}$} & \multirow{2}{*}{$\begin{array}{l}\text { Vaginal } \\
\mathrm{N} \\
\end{array}$} & \multirow{2}{*}{$\begin{array}{l}\text { Cesáreo } \\
\mathrm{N}\end{array}$} & \multirow{2}{*}{$\begin{array}{l}\text { Não informado } \\
\mathrm{N} \\
\end{array}$} & \multicolumn{2}{|l|}{ Total } \\
\hline & & & & $\mathrm{N}$ & $\%$ \\
\hline 10 a 14 & 2 & 13 & 0 & 15 & 0,5 \\
\hline 15 a 20 & 147 & 418 & 1 & 566 & 19,9 \\
\hline 21 a 30 & 223 & 1286 & 1 & 1510 & 53,1 \\
\hline 31 a 40 & 73 & 656 & 1 & 730 & 25,7 \\
\hline 41 a 50 & 2 & 23 & 0 & 25 & 0,9 \\
\hline Total & 447 & 2396 & 3 & 2846 & 100 \\
\hline Valor $\mathrm{P}^{1}$ & $<0,001$ & & & & \\
\hline \multicolumn{6}{|l|}{ Escolaridade } \\
\hline Nenhuma & 5 & 37 & 0 & 42 & 1,5 \\
\hline 1 a 3 anos & 19 & 42 & 0 & 61 & 2,1 \\
\hline 4 a 7 anos & 128 & 317 & 0 & 445 & 15,6 \\
\hline 8 a 11 anos & 273 & 1580 & 2 & 1855 & 65,2 \\
\hline 12 anos ou mais & 22 & 420 & 1 & 443 & 15,6 \\
\hline Total & & 2396 & 3 & 2846 & 100 \\
\hline Valor $\mathrm{P}^{1}$ & $<0,001$ & & & & \\
\hline \multicolumn{6}{|c|}{ Número de consultas de pré-natal } \\
\hline Nenhuma & 14 & 17 & 0 & 31 & 1,1 \\
\hline 1 a 3 & 25 & 43 & 0 & 68 & 2,4 \\
\hline 4 a 6 & 86 & 232 & 0 & 318 & 11,2 \\
\hline 7 ou mais & 322 & 2104 & 3 & 2429 & 85,3 \\
\hline Total & 447 & 2396 & 3 & 2846 & 100 \\
\hline Valor $\mathrm{P}^{1}$ & $<0,001$ & & & & \\
\hline \multicolumn{6}{|l|}{ Situação civil } \\
\hline Com companheiro & 228 & 1592 & 2 & 1822 & 64,0 \\
\hline Sem companheiro & 217 & 794 & 1 & 1012 & 35,6 \\
\hline Não informado & & 10 & 0 & 12 & 0,4 \\
\hline Total & 447 & 2396 & 3 & 2846 & 100 \\
\hline Valor $\mathrm{P}^{1}$ & $<0,001$ & & & & \\
\hline \multicolumn{6}{|l|}{ Etnia } \\
\hline Branca & 103 & 582 & 0 & 685 & 24,1 \\
\hline Não branca & 37 & 176 & 0 & 213 & 7,5 \\
\hline Não informada & 307 & 1638 & 3 & 1948 & 68,4 \\
\hline Total & 447 & 2396 & 3 & 2846 & 100 \\
\hline Valor $\mathrm{P}^{1}$ & 0,417 & & & & \\
\hline \multicolumn{6}{|l|}{ Peso do RN (gramas) } \\
\hline 0 a 999 & 11 & 19 & 0 & 30 & 1,5 \\
\hline 1000 a 1499 & 8 & 33 & 0 & 41 & 2,1 \\
\hline 1500 a 2499 & 50 & 260 & 0 & 310 & 15,6 \\
\hline 2500 a 2999 & 130 & 614 & 1 & 745 & 65,2 \\
\hline 3000 a 3999 & 241 & 1412 & 2 & 1655 & 15,6 \\
\hline 4000 ou mais & & 58 & 0 & 65 & \\
\hline Total & 447 & 2396 & 3 & 2846 & 100 \\
\hline Valor $\mathrm{P}^{1}$ & $<0,001$ & & & & \\
\hline \multicolumn{6}{|l|}{ Apgar 10. Minuto } \\
\hline 0 a 3 & 9 & 17 & 0 & 31 & 1,1 \\
\hline 4 a 7 & 50 & 43 & 0 & 68 & 2,4 \\
\hline 8 a 10 & 385 & 232 & 0 & 318 & 11,2 \\
\hline Não Informado & & 2104 & 3 & 2429 & 85,3 \\
\hline Total & 447 & 2396 & 3 & 2846 & 100 \\
\hline Valor $\mathrm{P}^{1}$ & $<0,001$ & & & & \\
\hline \multicolumn{6}{|l|}{ Apgar 5‥ Minuto } \\
\hline 0 a 3 & 6 & 6 & 0 & 12 & 1,1 \\
\hline 4 a 7 & 15 & 41 & 0 & 56 & 2,4 \\
\hline 8 a 10 & 424 & 2349 & 3 & 2776 & 11,2 \\
\hline Não Informado & 3 & 0 & 0 & 3 & 85,3 \\
\hline Total & 447 & 2396 & 3 & 2846 & 100 \\
\hline Valor $\mathrm{P}^{1}$ & $<0,001$ & & & & \\
\hline
\end{tabular}

${ }^{1}$ Valor $\mathrm{P}$ referente ao teste qui-quadrado a $\mathrm{P}<0,05$. 
Este estudo mostrou grande ocorrência da cesariana, corroborando pesquisas debatidas acerca da assistência obstétrica em nosso meio, que privilegia o parto cesáreo. Ao relacionar 0 tipo de parto com faixa etária da mãe, observou-se que a cesariana foi predominante em todas as faixas etárias. No caso de adolescentes e mulheres mais jovens, a via de parto por cesariana extrapola a indicação e a preferência das mulheres, comprometendo o futuro reprodutivo dessas mulheres, com a realização de sucessivas cesarianas $[15,16]$.

Mesmo com índice elevado de cesáreas em adolescentes, este estudo mostrou que o parto vaginal é maior em mães jovens e mães com idade superior optam pelo parto cesáreo. Dados também observados em outros estudos, que verificaram que as mulheres mais velhas tiveram maior probabilidade em realizar uma cesariana $[1,15]$.

Avaliando a associação entre tipo de parto e etnia, não houve significância $(P=0,417)$, mas deve-se atentar que em $68,4 \%$ não havia tal informação nos registros do SINASC e então não foi possível avaliar. Outros estudos mostram que mulheres brancas são mais submetidas a cesarianas que as de outra etnia $[16,17]$.

$\mathrm{Na}$ relação do tipo de parto com escolaridade, observou-se neste estudo que mães com menor escolaridade apresentaram maior ocorrência de parto vaginal, de modo que aquelas com maior escolaridade tiveram mais parto cesáreo. Tal fato foi evidenciado na pesquisa "Nascer no Brasil", revelando que mulheres brasileiras com menor escolaridade tiveram menos chances de realizar uma cesariana. Assim, a cesariana é mais realizada em mulheres brasileiras com maior padrão de escolaridade e de nível socioeconômico, além de usarem mais analgesia $[1,14,15]$.

Pode-se supor que alta escolaridade, maior idade, residir nas regiões mais ricas do país e ser de cor branca são proxys do uso de serviços privados, que apresentam altas taxas de cesariana quando comparados aos serviços públicos. No entanto, no Brasil, mesmo as usuárias dos serviços públicos também têm sido induzidas para a cesárea, muitas vezes buscando alternativas para financiamento de seu parto, em consonância com o médico de sua escolha [1].

Em relação ao número de consultas de pré-natal o resultado é semelhante, ou seja, neste estudo agrupamos tanto as mulheres assistidas no SUS quanto as de plano privado de saúde e em ambos os grupos as mulheres realizaram 7 ou mais consultas no pré-natal. $\mathrm{Na}$ análise estatística há tendência em considerar que mães com 4 a 6 consultas realizaram mais parto vaginal. Espera-se que as mulheres que tivessem mais consultas de pré-natal adequadamente conduzido fossem mais propensas ao parto normal/vaginal, mas em nosso estudo em qualquer situação a ocorrência da cesariana preponderou. $O$ maior acesso à saúde está relacionado a maiores intervenções. Assim, o que se verifica na região estudada é o que destacam alguns autores, ou seja, quanto maior o número de consultas maior a exposição à cesariana, relacionado principalmente as consultas e o parto serem realizadas pelo mesmo profissional médico $[16,18]$.

A qualidade precária dos serviços de saúde existentes, a falta de integração entre os cuidados de pré-natal e a assistência ao parto, bem como o aumento de cesarianas desnecessárias têm sido as principais justificativas para a elevada morbimortalidade materna e neonatal no Brasil, fato que está em descompasso com a evolução do acesso ao pré-natal e ao parto hospitalar [19]. O que tem sido debatido no Brasil é a pressa em provocar o nascimento das crianças, sem respeito a mulheres no processo de parturição. Ao contrário do esperado quando o pré-natal é adequado, desde o início da assistência na gestação a mulher/família já são "conduzidas" à cesárea e ter mais de 6 consultas de pré-natal não tem significado receberem informações sobre boas práticas e cuidados, muito pelo contrário [1,15,20].

O tipo de parto com relação ao estado civil das mulheres deste estudo evidenciou que a maioria das mães com companheiro optou pelo parto cesáreo, sugerindo que mulheres solteiras e sem companheiro são mais propensas a realizar parto normal. Tal resultado é mencionado por outros estudos que mulheres com nível socioeconômico mais alto são as que mais realizam cesarianas. Essas seriam as mulheres com maior acesso à saúde e menor risco gestacional e, assim, as taxas de cesáreas não têm como ser justificadas dentro das indicações obstétricas. Infelizmente, o que se vê no Brasil e particularmente nesse grupo, é que as mulheres com melhores condições de saúde materna e menor risco obstétrico são aquelas mais submetidas à cesariana.

Sobre o peso do RN associado ao tipo de parto observou-se nesta pesquisa que quanto menor o peso do RN, maior será a frequência da ocorrência de parto vaginal, o que é esperado, pois não há como indicar a cesárea em mulheres com idade gestacional menor e peso baixo do recém-nascido. 
O escore Apgar apresentou associação com o tipo de parto, mostrando que tanto 0 escore do $1^{\circ}$ e $5^{\circ}$ minuto foram superiores para os $\mathrm{RN}$ nascidos de parto cesáreo. Sendo assim, quando o RN apresenta um peso menor que $1000 \mathrm{~g}$, é esperado que tenha complicações relacionada à imaturidade que influenciam a nota do Apgar.

Neste estudo só analisamos as cesáreas e a maioria ocorreu com 39 semanas, portanto, com maturidade fetal. Os estudiosos advertem que os nascidos de cesárea eletiva e com hora marcada, sem entrar em trabalho de parto, apresentam risco elevado de morbidade respiratória leve e grave. É a morbidade neonatal near miss, que aumentava à medida que diminui a idade gestacional, mostrando que o trabalho de parto tem função importante na maturação pulmonar. Também aventam o uso de intervenções desnecessárias, que podem prejudicar tanto a mãe quanto o concepto [15]. A ausência dos mecanismos fisiológicos de adaptação ao nascimento implica risco para os bebês e impossibilitam o contato precoce pele a pele, início do aleitamento na primeira hora de vida [20].

Os dados obtidos neste estudo deixam em destaque a necessidade de proposição de ações e intervenções que minimizem a realização de cesáreas na microrregião sem indicação obstétrica. Sugerimos, como também é enfatizado em outros estudos, uma maior inserção de enfermeiros obstetras na condução do trabalho de parto, principalmente. Também, que os profissionais assistam as mulheres no ciclo gravídico puerperal baseando-se em diretrizes e protocolos assistenciais de órgãos internacionais e nacionais e contribuam para que a mulher participe ativamente de seu parto, proporcionando-lhe empoderamento para o protagonismo, no foco da humanização da assistência obstétrica.

Conclusão

Este estudo revelou que as mulheres estão sendo submetidas à cesariana de forma abusiva e até vergonhosa na microrregião pesquisada e que as características sociodemográficas interferem no tipo de parto, tornando-se como fator predisponente maior idade, maior escolaridade, ter companheiro, realizar pré-natal e o peso do RN ser indicativo para cesariana.

1. Rattner D, Moura EC. Nascimentos no Brasil: associação do tipo de parto com variáveis temporais e sociodemográficas. Rev Bras Saúde Matern Infant 2016;16(1):3947. https://doi.org/10.1590/1806-93042016000100005

2. Word Health Organization. Appropriate technology for birth. The Lancet 1985; 8452(2):436-7.

3. Brasil. MS. Secretaria de ciência, Tecnologia e Insumos Estratégicos. Comissão Nacional de Incorporação de Tecnologias no SUS. Diretrizes de atenção à gestante: a operação cesariana, 2016. [citado 2016 Jan 20]. Disponível em: http://conitec.gov.br

4. Cardoso JE, Barbosa RHS. O desencontro entre desejo e realidade: a indústria da cesariana entre mulheres de camadas médias no Rio de Janeiro, Brasil. Physis 2012;22(1):35-52.

5. Domingues RMSM, Dias MAB, Nakamura-Pereira M, Torres JA, d'Orsi E, Pereira APE, et al. Processo de decisão pelo tipo de parto no Brasil: da preferência inicial das mulheres à via de parto final. Cad Saúde Pública 2014;30Suppl 1:S101-16. https://doi.org/10.1590/0102-311X00105113

6. Rattner D, Rabello-Neto DL, Lansky S, Vilela MEA, Bastos MH. As cesarianas no Brasil: situação no ano de 2010, tendências e perspectivas. In: Brasil. Brasil 2011: uma análise da situação de saúde e a vigilância da saúde da mulher. Brasília: Ministério da Saúde; 2012. p.371-99.

7. WHO, UNICEF, UNFPA and The World Bank estimates. Geneva: World Health Organization, 2012. p. 59

8. Soler ZASG. CEPAHN - Centro de Preparo e Assistência Humanizados ao Nascimento: uma proposta para a região de São José do Rio Preto, São Paulo [livredocência]. São José do Rio Preto: Faculdade de Medicina de São José do Rio Preto; 2005.

9. Andrade MAC, Lima JBMCO. Modelo obstétrico e neonatal que defendemos e com o qual trabalhamos. Humanização do parto e do nascimento / Ministério da Saúde. Universidade Estadual do Ceará. Brasília: Ministério da Saúde; 2014. 465 p. 
10. Leal MC. Estar grávida no Brasil. Cad Saude Publica [Editorial] 2012;28(8):1420-21.

11. Leão MRC, Riesco MLG, Schneck CA, Angelo M. Reflexões sobre o excesso de cesariana no Brasil e autonomia das mulheres. Ciênc Saúde Coletiva 2013;18(8):2395400. https://doi.org/10.1590/S1413-81232013000800024

12. Torres JA. Cesariana e resultados neonatais em hospitais privados no Brasil: estudo comparativo de dois diferentes modelos de atenção perinatal. Cad Saúde Pública 2014;30(Supl):S220-S31. https://doi.org/10.1590/0102-311X00129813

13. Brasil. Ministério da Saúde. Secretaria de ciência, Tecnologia e Insumos Estratégicos. Departamento de Ciência e Tecnologia PNDS 2016 - Pesquisa Nacional de Demografia e Saúde da Mulher. Brasília: Ministério da Saúde; 2008,583p.

14. Domingues RMSM, Dias MAB, Nakamura-Pereira M, Torres JA, d'Orsi E, Pereira APE et al. Processo de decisão pelo tipo de parto no Brasil: da preferência inicial das mulheres à via de parto final. Cad Saúde Pública 2014;30Suppl 1:S101-16. https://doi.org/10.1590/0102-311X00105113

15. Leal MC, Domingues RMSM, Thema Filha MM, Dias MAB, Bastos MH et al. Intervenções obstétricas durante o trabalho de parto e parto em mulheres brasileiras de risco habitual. Cad Saúde Pública 2014;30(Supl):S17-S47. https://doi.org/10.1590/0102-311X00151513

16. Gama SGN, Pereira APE, Domingues RMSM, Theme Filha MM, Dias MAB, Bastos $\mathrm{MH}$. Fatores associados à cesariana entre primíparas adolescentes no Brasil, 20112012. Cad Saúde Pública 2014;30(Supl):S117-S27. https://doi.org/10.1590/0102$311 \times 00151513$

17. D'Orsi E, Bruggemann OM, Diniz CSG, Aguiar JM, Gusman CR, Torres JA, et al. Desigualdades sociais e satisfação das mulheres com o atendimento ao parto no Brasil: estudo nacional de base hospitalar. Cad Saúde Pública 2014;30 (Supl):S154S68. http://doi.org/10.1590/0102-311X00087813

18. Szwarcwald CL, Escalante JJC, Rabello Neto DL, Souza Júnior PRB, Victora CG. Estimação da razão de mortalidade materna no Brasil, 2008-2011. Cad Saúde Pública 2014;30(Supl):S71-S83. https://doi.org/10.1590/0102-311X00125313

19. Victora CG, Aquino EM, do Carmo Leal M, Monteiro CA, Barros FC, Szwarcwald CL. Maternal and childhealth in Brazil: progress and challenges. Lancet 2011;377:1863-76.

20. Chaves RL. O nascimento como experiência radical de mudança. Cad Saúde Pública 2014;30(Supl):S14-S16. 